\title{
Two-particle Momentum Correlation in Jets at the Tevatron
}

\author{
Sergo Jindariani \\ ( for CDF collaboration ) \\ Department of Physics, University of Florida \\ Gainesville, FL 32611, USA
}

\begin{abstract}
Presented are the measurements of two-particle momentum correlations in jets produced in p-pbar collisions at center of mass frame energy $1.96 \mathrm{TeV}$. Studies were performed for charged particles within a restricted opening angle of $0.5 \mathrm{rad}$ around the jet axis and for dijet events with various dijet masses. Comparison of the experimental results to the theoretical predictions obtained for partons within the framework of the resummed perturbative QCD ( Next-to-Leading Log Approximation) shows that the parton momentum correlations do survive the hadronization stage of jet fragmentation, thus, giving further support to the hypothesis of Local Parton-Hadron Duality.
\end{abstract}

Keywords: Fragmentation; jets; momentum correlations.

PACS: 12.38.-t; 12.38.Aw; 12.38.Bx; 12.38.Qk;

\section{INTRODUCTION}

Formation and evolution of jets is driven by emission of gluons at very small transverse momentum with respect to jet axis. Detailed studies of jet fragmentation allow one to investigate a fuzzy boundary between domains of perturbative QCD (pQCD) and non-perturbative hadronization. Theory sees fragmentation as a predominantly pQCD process. Analytical predictions are based on Next-to-LeadingLog Approximation (NLLA) ${ }^{1}$ calculations supplemented with the hypothesis of Local Parton-Hadron Duality (LPHD) ${ }^{2}$. Past measurements of particle multiplicity in jets and inclusive momentum distributions of particles in jets showed good agreement with theoretical predictions, suggesting that $\mathrm{pQCD}$ stage of jet formation must be dominant, and the role of non-perturbative stage is reduced to converting final partons to hadrons without significantly affecting the multiplicities and momenta. Momentum correlations, however, are more subtle effect and must be measured in order to conclude whether it survives the hadronization process.

In addition, fragmentation studies of jets at Tevatron complement measurements from e+e- and ep experiments providing a unique test of universality of jets. 


\section{MEASUREMENTS AND RESULTS}

CDF measurement of two-particle momentum correlation is based on $\sim 400 \mathrm{pb}^{-1}$ of dijet data and is made for a mixture of quark and gluon jets in the final state. The analysis is carried out in the dijet center-of-mass frame. Data were divided into seven subsamples with average dijet energy in subsample $M_{j j}=76,108148,200,270,360$ and $475 \mathrm{GeV}$. The correlation function is introduced in terms of variable $\xi=\log$ $\left(E_{\text {jel }} / P_{\text {hadron }}\right)$ and is measured as a ratio of two- and one-particle inclusive momentum distribution functions, i.e.

$$
\mathrm{C}\left(\xi_{1}, \xi_{2}\right)=\frac{\mathrm{D}\left(\xi_{1}, \xi_{2}\right)}{\mathrm{D}\left(\xi_{1}\right) \mathrm{D}\left(\xi_{2}\right)}
$$

where $\mathrm{D}\left(\xi_{1}, \xi_{2}\right)=\frac{\mathrm{d}^{2} \mathrm{~N}}{\mathrm{~d} \xi_{1} \mathrm{~d} \xi_{2}}$ and $\mathrm{D}(\xi)=\frac{\mathrm{dN}}{\mathrm{d} \xi}$ are normalized to unity.

Only charged particles in restricted cone with small opening angle of $\theta_{c}=0.5 \mathrm{rad}$ around jet axis were considered. Events with only one interaction were used in the sample. Contributions due to underlying event and other uncorrelated background were removed using the complementary cone technique ${ }^{3,4}$.

The theoretical prediction for correlation function ${ }^{5,6,7}$ is:

$$
\mathrm{C}\left(\Delta \xi_{1}, \Delta \xi_{2}\right)=\mathrm{C}_{0}+\mathrm{C}_{1}\left(\Delta \xi_{1}+\Delta \xi_{2}\right)+\mathrm{C}_{2}\left(\Delta \xi_{1}-\Delta \xi_{2}\right)^{2}
$$

where $\Delta \xi=\xi-\xi_{0}$ and $\xi_{0}$ - position of the peak of the inclusive particle momentum distribution in jets. Parameters $C_{0}, C_{1}$ and $C_{2}$ define strength of correlation and depend on a variable $\log \left(Q / Q_{e f f}\right)$ where $Q=E_{j e t} \theta_{c}$ is so called jet hardness and $Q_{\text {eff }}$ is parton shower cut-off scale used in theory.

Fig. 1 shows diagonal profiles of correlation distribution for subsample with $Q=50$ $\mathrm{GeV}$. Also shown on Fig. 1 is fit to CDF data using theoretical function and theoretical function for $Q_{e f f}=230 \mathrm{MeV}$ (dashed line). Smaller error bars correspond to only statistical uncertainty while larger ones to total uncertainty - statistical and systematic uncertainty added in quadrature. Shaded area represents uncertainty of the fit. One can see that trends in data and theory are the same - there's enhanced chance of finding two particles with the same value of momentum and this effect becomes larger for softer particles. The comparison to Monte-Carlo reveals that Pythia Tune A and Herwig 6.5 reproduce correlation fairly well.

Fig.2 shows evolution of parameters $C_{1}$ and $C_{2}$ as a function of jet hardness $Q$. Each data point corresponds to the value of parameter measured in particular dijet bin. CDF data points are fit to analytical NLLA function, while parton shower cut-off scale $Q_{\text {eff }}$ is treated as free parameter. Theoretical curves for samples with only quark jets and only gluon jets in the final state are also shown. The value of $Q_{\text {eff }}$ obtained from the fit of $C_{1}$ is $\sim 147 \pm 10$ (stat) \pm 79 (syst) $M e V$. The value of $Q_{\text {eff }}$ obtained from the fit of $C_{2}$ is $\sim 131 \pm 12$ (stat) \pm 86 (syst) $\mathrm{MeV}$. 

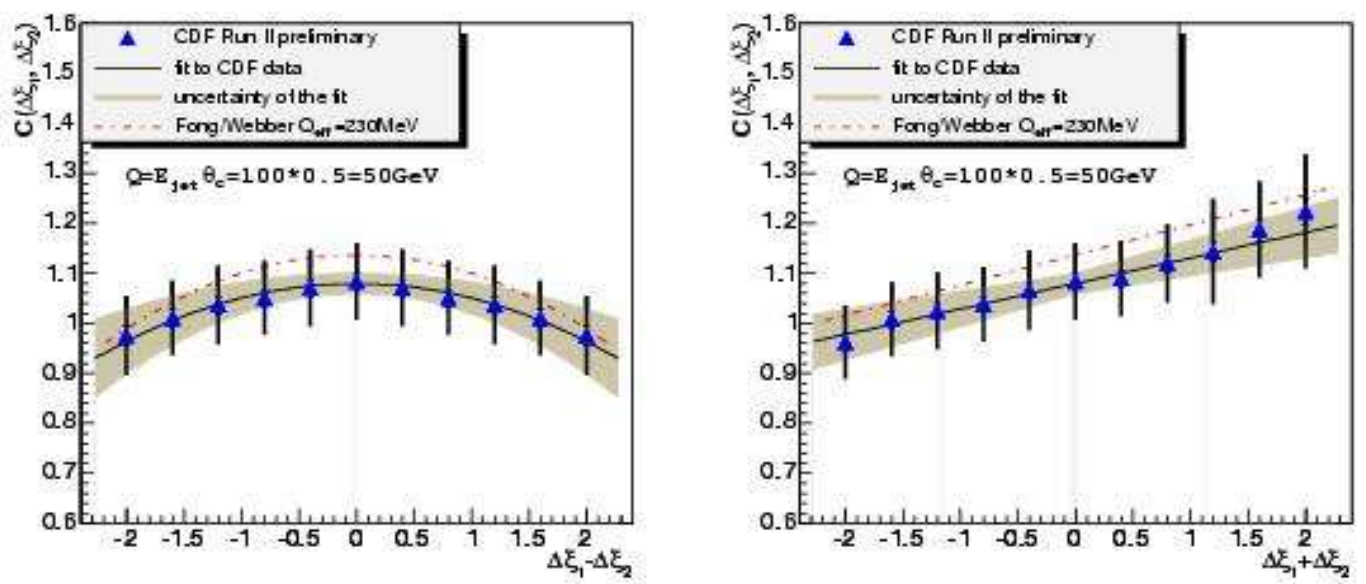

FIGURE 1. Diagonal profiles $\Delta \xi_{1}=-\Delta \xi_{2}$ and $\Delta \xi_{1}=\Delta \xi_{2}$ of correlation distribution for bin with $Q=50$ $\mathrm{GeV}$. Comparison of CDF results to analytical NLLA calculation.
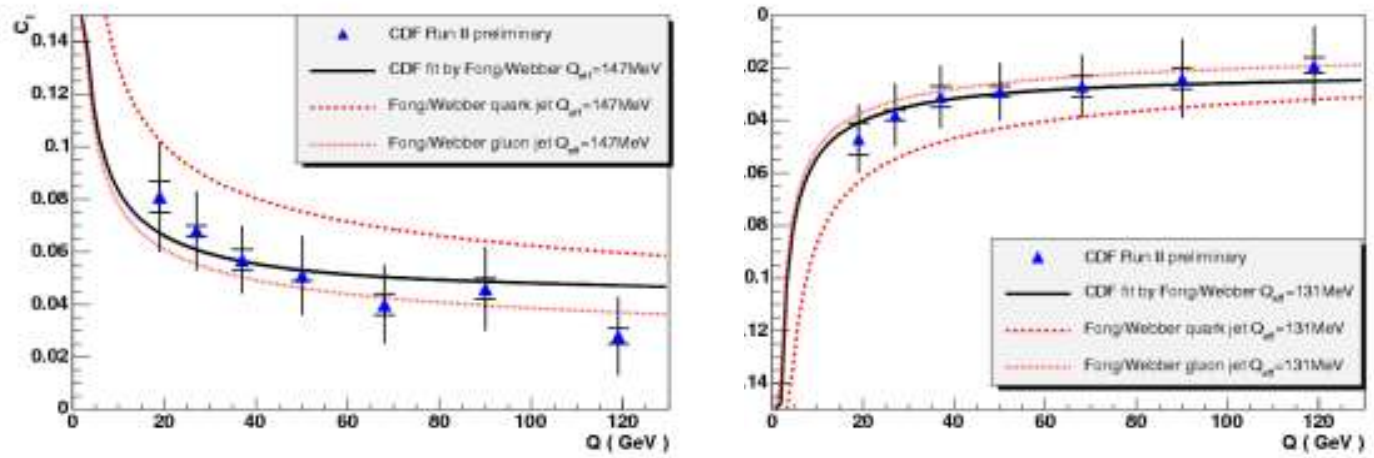

FIGURE 2. Evolution of correlation parameters $C_{1}$ and $C_{2}$ with jet hardness scale $Q$. Comparison of CDF data to analytical NLLA curves.

\section{CONCLUSIONS}

CDF has studied two-particle momentum correlation in jets. The results are found to be in agreement with re-summed NLLA calculations. The values of the parton shower cut-off scale extracted from the dependence of $C_{1}$ and $C_{2}$ on energy scale are self-consistent and reasonable. Correlation clearly survives hadronization process, thus, giving further support to the hypothesis of LPHD.

\section{REFERENCES}

1. Yu.Dokshitzer, V.Khoze, A.Mueller and S.Troyan, Basics of perturbative QCD, ed. J.Tran Thanh Van ( Editions Frontieres, Gif-sur-Yvette, 1991).

2. Ya. I. Azimov, Yu.Dokshitzer, V.Khoze and S.Troyan, Z. Phys., C27, 65 (1985); 31, 213 (1986).

3. CDF Collab.: T.Affolder et al., Phys.Rev. Letters 87, 211804 (2001).

4. CDF Collab.: T.Acosta et al., Phys.Rev. D68, 012003 (2003).

5. C. Fong and B.R. Webber, Phys.Lett. B229, 289 (1989).

6. C. Fong and B.R. Webber, Phys.Lett. B241, 255 (1989).

7. C. Fong and B.R. Webber, Nucl.Phys. B355, 54 (1991). 